MPI-PhT/96-25

hep-th/9604161

\title{
Perturbative versus Non-perturbative QFT Lessons from the $O(3)$ NLS Model
}

\author{
J. Balog®, M. Niedermaier \\ Max-Planck-Institut für Physik \\ - Werner Heisenberg Institut - \\ Föhringer Ring 6, 80805 Munich, Germany \\ T. Hauer \\ Center for Theoretical Physics, Laboratory for Nuclear Science \\ Massachusetts Institute of Technology \\ Cambridge, MA 02139, USA
}

\begin{abstract}
The two-point functions of the energy-momentum tensor and the Noether current are used to probe the $O(3)$ nonlinear sigma model in an energy range below $10^{4}$ in units of the mass gap $m$. We argue that the form factor approach, with the form factor series trunctated at the 6-particle level, provides an almost exact solution of the model in this energy range. The onset of the (2-loop) perturbative regime is found to occur only at energies around $100 \mathrm{~m}$.
\end{abstract}

*On leave of absence from the Central Research Institute for Physics, Budapest, Hungary 


\section{Introduction}

The $O(3)$ Nonlinear Sigma (NLS) model has long been appreciated as a 2-dimensional testing ground for nonabelian gauge theories. In particular it shares features like dynamical mass generation, running coupling constant and (conjectured) asymptotic freedom with its 4-dimensional cousins. A central issue is therefore to determine the domain of validity of perturbation theory in the model. This requires an approach which is capable of describing the system both in the non-perturbative low energy and the perturbative high energy region. Although it could not have been expected from the outset, the form factor approach [1, 2] (which essentially provides a low energy expansion of $n$-point functions based on the exact $S$-matrix [3]) turns out to be well suited for that purpose. Using the two-point functions of the energy-momentum tensor and the Noether current to probe the system, we find that the form factor series truncated at the 6-particle level provides an almost exact solution of the model in the energy range below $10^{4}$ in units of the mass gap $m$. A similar fast convergence of the low energy expansion has previously been observed for the class of models with diagonal $S$-matrices [4, 5, 6] .

We compare our results with Monte Carlo data [7] and 2-loop perturbation theory (PT). Within the range considered 2-loop PT appears to yield an accurate (within one percent) description of the system only for energies above $100 \mathrm{~m}$, provided one uses the known exact value of the Lambda parameter [8] to fix its absolute normalization. In 4-dimensional gauge theories of course neither the exact Lambda parameter nor the (almost) exact form factor curve is available. Based on the low energy Monte Carlo data alone one might therefore be tempted to maximize the apparent domain of validity of PT by tuning the Lambda parameter such as to match the relevant part of the Monte Carlo data. Doing this in the NLS model would give a value for the Lambda parameter that is smaller than the exact value by about $10 \%$. This emphasizes the importance to have an independent estimate for the onset of the (2-loop) perturbative regime.

\section{Spectral representation of two point functions}

The form factors characterize an (integrable as well as non-integrable) QFT in a similar way as the $n$-point functions do. Assuming the existence of a resolution of the identity in terms of asymptotic multi-particle states, the $n$-point functions can in principle be recovered from the form factors. In the important case of the two-point functions this amounts to the well-known spectral representation. Explicitly for the Minkowski two- 
point function (Wightman function) of some local operator $\mathcal{O}$ one obtains in a first step

$$
W^{\mathcal{O}}(x-y)=\sum_{n \geq 1} \frac{1}{n !} \int \prod_{j=1}^{n} \frac{d \theta_{j}}{4 \pi} e^{-i\left(x^{0}-y^{0}\right) P_{0}^{(n)}(\theta)-i\left(x^{1}-y^{1}\right) P_{1}^{(n)}(\theta)}\left|f^{(\mathcal{O}, n)}(\theta)\right|^{2}
$$

where $f^{(\mathcal{O}, n)}(\theta)=\left\langle\operatorname{vac}|\mathcal{O}(0)| \theta_{n}, \ldots, \theta_{1}\right\rangle$ are the form factors of $\mathcal{O}$ and $P_{\mu}^{(n)}(\theta)=\sum_{i} p_{\mu}\left(\theta_{i}\right)$, $p_{0}(\theta)=m \operatorname{ch} \theta, p_{1}(\theta)=m \operatorname{sh} \theta$ are the eigenvalues of energy and momentum on an $n$-particle state. The local operators are classified by various quantum numbers, in particular by their Lorentz spin $s$ and their mass dimension $\Delta$. The form factors of a local operator with quantum numbers $(\Delta, s),|s| \leq \Delta$ can be assumed to be of the form

$$
f^{(n, \mathcal{O})}(\theta)=i^{s}\left(\frac{m}{\sqrt{2}} \sum_{j} e^{\theta_{j}}\right)^{\frac{\Delta+s}{2}}\left(\frac{m}{\sqrt{2}} \sum_{j} e^{-\theta_{j}}\right)^{\frac{\Delta-s}{2}} f^{(n)}(\theta),
$$

where $f^{(n)}(\theta)$ carries quantum numbers $(\Delta, s)=(0,0)$ and is a function of the rapidity differences only. We shall henceforth always work with the 'scalarized' form factors $f^{(n)}(\theta)$ and drop the superscript ' $\mathcal{O}$ '. For many purposes it is useful to rewrite (1) in the form of a Källen-Lehmann spectral representation. Changing integration variables according to

$$
\begin{aligned}
& u_{i}=\theta_{i}-\theta_{i+1}, \quad 1 \leq i \leq n-1, \quad \alpha=\ln \left(\frac{m\left(e^{\theta_{1}}+\ldots+e^{\theta_{n}}\right)}{M^{(n)}(u)}\right), \\
& M^{(n)}(u)^{2}=m^{2}\left[n+2 \sum_{i<j} \operatorname{ch}\left(u_{i}+\ldots+u_{j-1}\right)\right]
\end{aligned}
$$

and considering the case of an operator $\mathcal{O}$ with quantum numbers $(\Delta, s)$ one obtains

$$
\begin{aligned}
& W^{\mathcal{O}}(x-y)=-i \int_{0}^{\infty} d \mu \rho(\mu) D^{(\Delta, s)}(x-y ; \mu), \\
& \rho(\mu)=\sum_{n=1}^{[\mu / m]} \rho^{(n)}(\mu), \quad \rho^{(1)}(\mu)=\delta(\mu-m)\left|f^{(1)}\right|^{2}, \\
& \rho^{(n)}(\mu)=\int_{0}^{\infty} \frac{d u_{1} \ldots d u_{n-1}}{(4 \pi)^{n-1}}\left|f^{(n)}(u)\right|^{2} \delta\left(\mu-M^{(n)}(u)\right), \quad n \geq 2 .
\end{aligned}
$$

Notice that no problem of convergence arises for the spectral density. First, each $n$ particle contribution exists because, for fixed $\mu$, the integrand has support only in a

\footnotetext{
${ }^{1}$ Our kinematical conventions are: $\left(x^{0}, x^{1}\right)$ are coordinates on 2-dimensional Minkowski space $\mathbb{R}^{1,1}$ with bilinear form $x \cdot y=x^{\mu} \eta_{\mu \nu} y^{\nu}, \eta=\operatorname{diag}(1,-1)$. Lightcone coordinates are $x^{ \pm}=\left(x^{0} \pm x^{1}\right) / \sqrt{2}=x_{\mp}$. The normalization of the 1-particle states is $\left\langle\theta_{1} \mid \theta_{2}\right\rangle=4 \pi \delta\left(\theta_{1}-\theta_{2}\right)$, which corresponds to the standard normalization in $d+1$ dimensions, specialized to $d=1$. For simplicity we suppress internal indices for most of this section.
} 
compact domain $V(\mu) \subset \mathbb{R}_{+}^{n-1}$ in which the form factors are bounded functions, so that the integration is well-defined. Viewed as a function of $\mu$ one observes that $\rho^{(n)}(\mu)$ has support only for $\mu \geq m n$. Therefore only a finite number of terms (those with $n \leq[\mu / m]$, $[x]$ being the integer part of $x$ ) contribute to $\rho(\mu)$. Under some mild assumptions on the growth of $\rho(\mu)$ the existence of the spectral density then guarantees that of the 2-point function, as defined through (3). The integration kernel $D^{(\Delta, s)}(x ; m)$ in $(3)$ is given by

$$
\begin{aligned}
D^{(\Delta, s)}(x ; m) & =i \int \frac{d^{2} p}{2 \pi} \theta\left(p_{0}\right) \delta\left(p^{2}-m^{2}\right)\left(p_{+}\right)^{\Delta+s}\left(p_{-}\right)^{\Delta-s} e^{-i p \cdot x} \\
& =\left(i \partial_{+}\right)^{\Delta+s}\left(i \partial_{-}\right)^{\Delta-s} D(x ; m)
\end{aligned}
$$

where $D(x ; m):=D^{(0,0)}(x ; m)$ is the two-point function of a free scalar field of mass $m$. (In general however $D^{(\Delta, s)}(x ; m)$ does not coincide with the two-point function of some composite operator $\mathcal{O}$ built from the scalar field). In the spinless case one obtains from the wave equation $D^{(\Delta, 0)}(x ; m)=\left(m^{2} / 2\right)^{\Delta} D(x ; m)$. The support properties of the kernel functions can be extracted by evaluating them in terms of Bessel functions; in particular for strictly spacelike distances one finds

$$
D^{(\Delta, s)}(x ; m)=i\left(\frac{m^{2}}{2}\right)^{\Delta} \frac{1}{2 \pi}\left(\frac{x^{-}}{x^{+}}\right)^{s} K_{2 s}\left(m \sqrt{-x^{2}}\right), \quad x^{2}<0 .
$$

Essentially this also yields the kernel function entering the spectral representation of the Euclidean two-point function (Schwinger function). The latter can be defined by $S^{\mathcal{O}}\left(x_{1}, x_{2}\right):=W^{\mathcal{O}}\left(-i x_{2}, x_{1}\right)$ for $x_{2}>0$ and then by analytic continuation to $x_{2}<0$. In the spectral representation (3) this yields $-i D^{(\Delta, s)}\left(-i x_{2}, x_{1} ; m\right)$ for the Euclidean kernel function with the right hand of $(5)$ taken for $D^{(\Delta, s)}(x ; m)$. Notice that the spectral density is the same in the Minkowski and in the Euclidean case. Return now to the Minkowski space situation. For comparison with perturbation theory one needs the time-ordered two-point function and its Fourier transform. Its spectral representation is easily read off from (3), (4)

$$
\begin{aligned}
G^{\mathcal{O}}(x-y) & =-i \int_{0}^{\infty} d \mu \rho(\mu) G^{(\Delta, s)}(x-y ; \mu) \\
G^{(\Delta, s)}(x ; m) & =\theta\left(x_{0}\right) D^{(\Delta, s)}(x ; m)-\theta\left(-x_{0}\right)\left[D^{(\Delta, s)}(x ; m)\right]^{*} \\
& =\int \frac{d^{2} p}{(2 \pi)^{2}} e^{-i p \cdot x} \frac{\left(p_{+}\right)^{\Delta+s}\left(p_{-}\right)^{\Delta-s}}{p^{2}-m^{2}+i \epsilon}
\end{aligned}
$$

For the Fourier transform this means

$$
\widetilde{G}^{\mathcal{O}}(p)=i\left(-\frac{1}{2}\right)^{\Delta}\left(\frac{p_{+}}{p_{-}}\right)^{s} I\left(-p^{2}\right), \quad I(z)=z^{\Delta} \int_{0}^{\infty} d \mu \frac{\rho(\mu)}{z+\mu^{2}-i \epsilon} .
$$


The definition of $I(z)$ was chosen such that it has a cut along the negative real axis and one can recover the spectral density from the discontinuity along this cut.

As an example consider the case of the energy-momentum (EM) tensor. It is of additional interest, because its spectral density is closely related to the Zamolodchikov C-function [9]. The spectral representation of the Minkowski 2-point function is

$$
\begin{aligned}
& W_{\mu \nu, \rho \sigma}^{E M}(x-y)=-i \int_{0}^{\infty} d \mu \rho(\mu) D_{\mu \nu, \rho \sigma}(x-y ; \mu) \\
& D_{\mu \nu, \rho \sigma}(x ; m)=i \int \frac{d^{2} p}{2 \pi} \theta\left(p_{0}\right) \delta\left(p^{2}-m^{2}\right) e^{-i p \cdot x}\left(\eta_{\mu \nu} p^{2}-p_{\mu} p_{\nu}\right)\left(\eta_{\rho \sigma} p^{2}-p_{\rho} p_{\sigma}\right) .
\end{aligned}
$$

The lightcone components correspond to the $S O(1,1)$-irreducible pieces; in particular $D_{++,++}(x ; m)$ and $D_{+-,+-}(x ; m)$ coincide with $D^{(2,2)}(x ; m)$ and $D^{(2,0)}(x ; m)$, respectively. Combining (3) and (5) one finds for the behaviour at small spacelike distances

$$
\begin{aligned}
W_{++,++}^{E M}(x) & =\left(\frac{x^{-}}{x^{+}}\right)^{2}\left[\frac{c}{2 \pi^{2}} \frac{1}{\left(x^{2}\right)^{2}}+O\left(\frac{1}{x^{2}}\right)\right], \quad x^{2}<0, \\
c & =12 \pi \int_{0}^{\infty} d \mu \rho(\mu) .
\end{aligned}
$$

The number $c$ as defined here coincides with the central charge of the Virasoro algebra in the conformal field theory describing the UV fixed point of the renormalization group. This latter fact is part of the statement of Zamolodchikov's C-theorem. The proof of the C-theorem is particularly transparent from the viewpoint of the spectral representation [10]. The normalization is such that $H=\int_{-\infty}^{\infty} d x^{1} T_{00}(x)$ has eigenvalue $\sqrt{p^{2}+m^{2}}$ on an asymptotic single particle state, where $m$ is the mass gap. For the 2-particle form factor of the $T_{+-}$component this implies

$$
f^{\left(T_{+-}, 2\right)}(\theta+i \pi, \theta)=m^{2}
$$

which fixes the normalization of all higher particle EM form factors. The scalarized form factors of all components can be interpreted as the form factors of a single field $\tau$ defined by $T_{\mu \nu}=\epsilon_{\mu \rho} \epsilon_{\nu \sigma} \partial^{\rho} \partial^{\sigma} \tau$. The conservation equation is then built into the parametrization (2).

Also a Noether current comes with an intrinsic normalization, which arises from the Lie algebra of its conserved charges. In the case of the $O(3)$ NLS model one has $\left[Q_{a}, Q_{b}\right]=$ $i \epsilon_{a b c} Q_{c}$ and

$$
Q_{a}|\theta\rangle_{b}=i \epsilon_{a b c}|\theta\rangle_{c},
$$

\footnotetext{
${ }^{2}$ In particular $\mathrm{c}$ is finite; for other operators the integral over the spectral density will in general not converge.
} 
where $Q_{a}=\int d x^{1} J_{0, a}(x)$ is the conserved charge of the $O(3)$ Noether current. For the 2-particle form factor of $J_{0, a}$ this implies

$$
f_{b c}^{J_{0, a}}(\theta+i \pi, \theta)=-2 i m \epsilon_{a b c} \operatorname{ch} \theta
$$

which fixes the normalization of all higher current form factors. The scalarized form factors of both (spacetime) components can be interpreted as the form factors of the field $\tau_{a}$ defined through $J_{\mu, a}=\epsilon_{\mu}{ }^{\nu} \partial_{\nu} \tau_{a}$. The two point function of $J_{\mu, a}(x)$ and $J_{\nu, b}(y)$ and hence the spectral density is of course proportional to $\delta_{a b}$.

\section{Polynomial $O(3)$-irreducible form factors}

Solutions of the form factor equations with the $O(3)$-invariant $S$-matrix [3] have been obtained by Kirillov and Smirnov [1] by a fusing procedure from the previously known form factors of the $S U(2)$ Thirring model [2]. Unfortunately these solutions refer to a basis in the tensor product $\mathbf{3}^{\otimes n}(\mathbf{2 l}+\mathbf{1}, l \in \mathbb{N}$ being the $O(3)$ irreps of spin $l)$ that is related to the canonical basis through a complicated rapidity-dependent basis transformation. Since the most interesting local operators in the model all transform irreducibly under $O(3)$ one has to determine the intertwiners

$$
Q_{l}^{(n)}: 3^{\otimes n} \longrightarrow 2 \mathbf{l}+\mathbf{1}
$$

to the canonical basis in $\mathbb{C}^{2 l+1} \cong \mathbf{2 l}+\mathbf{1}$. If one starts from the Kirillov-Smirnov basis in $\mathbf{3}^{\otimes n}$ also these intertwiners will be rapidity-dependent and their explicit computation is almost as difficult as the computation of the form factors themselves. We therefore found it easier to start afresh and to work with the canonical basis in $\mathbf{3}^{\otimes n}$ and constant intertwiners. A detailed exposition of the technique and the results will be given elsewhere 12 .

In survey we studied the following four local operators, which seem to be the most interesting ones in the $O(3)$ model: The 'fundamental' spin field $S$, the Noether current, the EM tensor and the topological charge (TC) density. For orientation we tabulate the expressions in terms of the field $S$, their quantum numbers $(l, \Delta)$ and the particle numbers of the form factors computed. 


\begin{tabular}{c|c|c|c}
$\mathcal{O}$ & $\mathcal{O}[S]$ & $(l, \Delta)$ & ff's computed \\
\hline spin & $S$ & $(1,0)$ & $1,3,(5)$ \\
current & $j_{\mu}=S \times \partial_{\mu} S$ & $(1,1)$ & $2,4,(6)$ \\
EM tensor & $T_{\mu \nu}=\partial_{\mu} S \cdot \partial_{\nu} S-\frac{1}{2} \eta_{\mu \nu} \partial^{\rho} S \cdot \partial_{\rho} S$ & $(0,2)$ & $2,4,(6)$ \\
TC density & $q=S \cdot\left(\partial_{\mu} S \times \partial_{\nu} S\right) \epsilon_{\mu \nu}$ & $(0,2)$ & $3,(5)$
\end{tabular}

The explicit formulae can be found in [12]. The brackets around the last particle number indicate that the resulting expressions for the form factors are too long to be communicated in print; instead we shall give the expressions for their modulus squares, which is also the quantitiy entering the spectral densities. Higher particle contributions could be computed in principle, although more refined techniques would be necessarry to circumvent limitations in computer power. It seems however that higher particle contributions to the spectral density are strongly suppressed in the $O(3)$-model. Indeed, in section 4 we shall argue that truncating the form factor series at the 5 or 6 particle level provides an 'almost exact' solution of the model up to energies of about $10^{4}$ times the mass gap. 'Almost exact' means that within this energy range the deviation of the true spectral densities from the truncated ones is estimated to be less than a percent. If one accepts the conventional wisdom about the asymptotic freedom of the model at very high energies, the combination of the non-perturbative form factor results (for energies up to $10^{4}$ in units of the mass gap) and the perturbative expansion at energies above $10^{4}$ would provide a solution of the model sufficient for most practical purposes. For issues of principle it would nevertheless be highly desirable to get some control on the higher particle contributions, in particular because the 'conventional wisdom' has been challenged [13]. Some first results in this direction will also be presented in [12]. An important such issue is trying to actually prove (or disprove) asymptotic freedom.

We shall not discuss the construction of the form factors here. Let us just mention the decomposition into $O(3)$ irreducible components. Let $f_{l, A}(\theta)$ denote the component of $f_{A}(\theta)$ in $\mathbf{2 l}+\mathbf{1}$. Explicitly $f_{l, A}(\theta)$ is an $n$-index $O(3)$ tensor parametrized by $m_{l}(n)$ independent functions, where $m_{l}(n)$ is the multiplicity with which $\mathbf{2 l}+\mathbf{1}$ occurs in $\mathbf{3}^{\otimes n}$. The choice of a parametrization amounts to a choice of an intertwiner $Q_{l}$ between the $O(3)$ representations $\mathbf{3}^{\otimes n}$ and $\mathbf{2 l}+\mathbf{1}$. Picking a basis in both spaces one can write

$$
f_{l, A}(\theta)=Q_{l, A}^{\alpha} g_{\alpha}(\theta) .
$$

\footnotetext{
${ }^{3}$ The difference in size is enormous. The 6-particle form factor of the current has about 3.4 Mbytes, i.e. approx. 700 A4 pages; the most compact form of the square requires less than one page.
} 
As remarked before, in contrast to [11], we work with the canonical basis in $\mathbf{3}^{\otimes n}$ and constant (rapidity-independent) intertwiners $Q_{l, A}$. (This means that each component $Q_{l, A}^{\alpha}$ can explicitly be written as a combination of antisymmetric epsilon tensors and Kronecker deltas.) For the components $g_{\alpha}(\theta)$ we used the following Ansatz

$$
\begin{aligned}
& g_{\alpha}(\theta)=\bar{g}_{\alpha}(\theta) \pi^{3(n-2) / 2} \prod_{k>r} \psi\left(\theta_{k}-\theta_{r}\right), \quad 1 \leq \alpha \leq m_{l}(n) \\
& \psi(\theta)=\frac{\theta-i \pi}{\theta(2 \pi i-\theta)} \tanh ^{2}(\theta / 2) .
\end{aligned}
$$

The main structural result can then be summarized as follows [14, 15, 12]:

Fact: The form factor equations for the $O(3)$ NLS model decompose into decoupled recursive systems for the $O(3)$-irreducible components $g^{(n)}(\theta)=\left(g_{1}, \ldots, g_{m_{l}(n)}\right)(\theta)$. There exist unique sequences of solutions $\left(g^{(n)}(\theta)\right)_{n \geq 1}$ of the form $(12)$ s.t. $\bar{g}^{(n)}(\theta)=\left(\bar{g}_{1}, \ldots, \bar{g}_{m_{l}(n)}\right)(\theta)$ are polynomials in the rapidities of total degree $N=\frac{1}{2}\left(n^{2}-3 n\right)+N_{0}$ and partial degree $p=n-2+p_{0}$.

Here 'partial degree' means the degree in an individual variable. For the operators considered before the initial values are given by $\left(N_{0}, p_{0}\right)=(0,-1)$ for EM tensor \& TC density and $\left(N_{0}, p_{0}\right)=(1,0)$ for Current \& Spin. The fact that the form factors can be reduced to polynomial expressions is specific for the $O(3)$ model and will cease to hold for the $O(N)$ NLS models with $N>3$. The first part of the above statement in particular means that the $O(3)$ spin is a good quantum number for form factor sequences: If one member of the sequence is $O(3)$-irreducible of spin $l$ then all other members will be too. This is only seemingly trivial; it will not hold for any Lie group and any $S$-matrix.

For the computation of the spectral densities and the two point functions the modulus square of the form factors is needed. A version of $\left|f_{l}^{(n)}(\theta)\right|^{2}$ that allows analytic continuation to complex rapidities is

$$
\begin{gathered}
f_{l, a_{n} \ldots a_{1}}\left(\theta_{n}, \ldots, \theta_{1}\right) f_{l, a_{1} \ldots a_{n}}\left(\theta_{1}, \ldots, \theta_{n}\right)=g_{\alpha}\left(\theta_{n}, \ldots, \theta_{1}\right) C_{\alpha, \beta} g_{\beta}\left(\theta_{1}, \ldots, \theta_{n}\right) \\
=C_{l} \frac{1}{4} \pi^{3 n-2} \bar{G}_{l}^{(n)}(\theta) \prod_{k>r} \psi\left(\theta_{k}-\theta_{r}\right) \prod_{i<j} \psi\left(\theta_{i}-\theta_{j}\right)
\end{gathered}
$$

where $C_{\alpha, \beta}$ is a constant 'metric' and $l=0,1$ correspond to the EM tensor \& TC density and Current \& Spin series, respectively. The prefactor $C_{l}$ is given by $C_{0}=3$ and $C_{1}=\delta_{a b}$. The so defined square is a completely symmetric function of the rapidities; in particular its polynomial part $\bar{G}_{l}^{(n)}(\theta)$ is a completely symmetric, boost invariant polynomial in the rapidities. (It depends only on the rapidity differences.) By choosing an appropriate basis 
in the space of symmetric polynomials it can be brought into a very compact form, as compared with what one might guess from the size of the form factors (c.f. footnote 3 ). A convenient basis is obtained as follows: For fixed $n$ let $\sigma_{1}^{(n)}, \ldots, \sigma_{n}^{(n)}$ denote the elementary symmetric polynomials in $\theta_{1}, \ldots, \theta_{n}$, i.e.

$$
\sigma_{k}^{(n)}=\sum_{i_{1}<\ldots<i_{k}} \theta_{i_{1}} \ldots \theta_{i_{k}}
$$

Then define polynomials $\tau_{k}^{(n)}$ by

$$
\begin{aligned}
& \tau_{k}^{(n)}\left(\theta_{1}, \ldots, \theta_{n}\right)=\sigma_{k}^{(n)}\left(\widehat{\theta}_{1}, \ldots, \widehat{\theta}_{n}\right), \quad 2 \leq k \leq n, \\
& \tau_{1}^{(n)}=\frac{1}{n} \sigma_{1}^{(n)}, \quad \widehat{\theta}_{j}=\theta_{j}-\frac{1}{n}\left(\theta_{1}+\ldots+\theta_{n}\right) .
\end{aligned}
$$

All monomials in the $\tau_{k}^{(n)}, k \geq 2$ are manifestly boost invariant. (The price to pay is that the partial degree is no longer manifest.) For the sake of illustration we display the polynomial part of the 4-particle squares of the EM tensor and the Current in this basis (where the superscripts '(4)' are suppressed):

EM tensor:

$\bar{G}_{0}^{(4)}(\theta)=4\left(\tau_{2}^{2}+12 \tau_{4}\right)-\pi^{2} 32 \tau_{2}+28 \pi^{4}$,

Current:

$$
\bar{G}_{1}^{(4)}(\theta)=-4\left(6 \tau_{2}^{3}+9 \tau_{3}^{2}+40 \tau_{2} \tau_{4}\right)+8 \pi^{2}\left(25 \tau_{2}^{2}+44 \tau_{4}\right)-448 \pi^{4} \tau_{2}+272 \pi^{6} .
$$

\section{Results for spectral densities and two-point functions}

Knowing the form factor squares the evaluation of the spectral densities and the two-point functions is in principle straightforward. The integrations in (3) can be done numerically to good accuracy. Here we shall restrict attention to the EM tensor and the Noether current. For the spectral densities an accuracy of $10^{-3}$ was used and the results for the EM tensor and the Noether current are shown in figures 1 and 2, respectively. In the EM case one can use equation (8) to compute the $n$-particle contributions to the central charge. One finds

$$
c^{(2)}=1.603, \quad c^{(4)}=0.194, \quad c^{(6)}=0.072, \quad c^{(n)}=12 \pi \int_{0}^{\infty} d \mu \rho^{(n)}(\mu)
$$


If one accepts asymptotic freedom as a working hypthesis, (tree level) PT predicts $c=2$, corresponding to the two unconstrained bosonic degrees of freedom. The form factor computation shows that this is compatible with the non-perturbative low energy dynamics of the model and that even for an extreme UV quantity like the central charge the low particle contributions dominate. An alternative non-perturbative consistency check is provided by the thermodynamic Bethe Ansatz, which also yields $c=2$ [16]. Let us point out that the value $c=2$ does not carry much information about the nature of the UV limiting CFT. For a number of reasons it cannot be that of two decoupled free bosons.

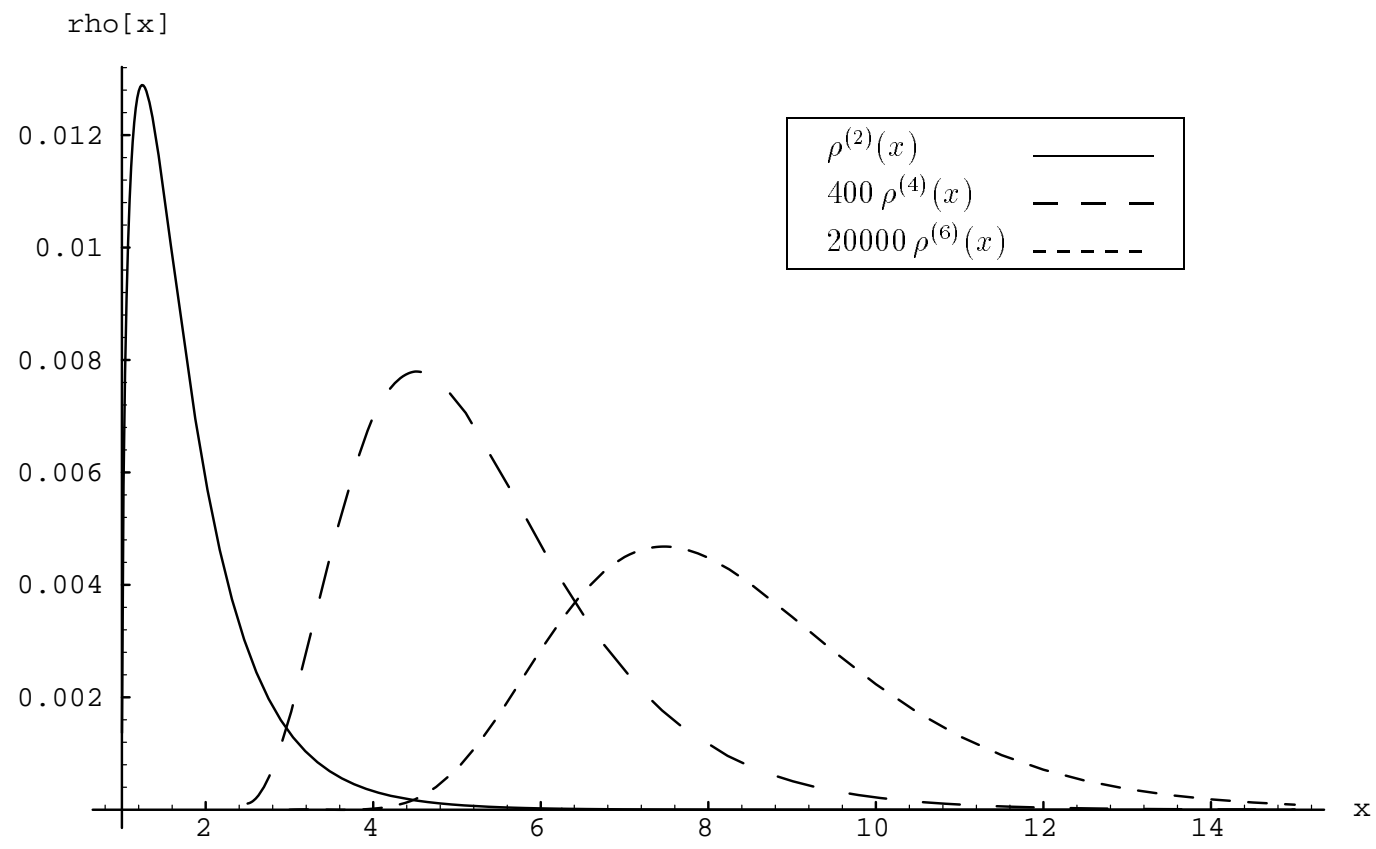

Figure 1: 2-, 4-, and 6-particle contribution to the spectral density of the EM tensor as a function of $x=\log _{2}(\mu / m)$.

Figures 1 and 2 illustrate some general features of the $n$-particle contributions to the spectral densities. First one observes a strong suppression of the higher particle contributions, as far as the value of the maximum and the enclosed area are concerned. Generally speaking the maximum of $\rho^{(n)}(\mu)$ is smaller by 1.5 to 2.5 orders of magnitudes compared to the maximum of $\rho^{(n-2)}(\mu)$, while the position of the maximum is shifted towards higher energies. Nevertheless at sufficiently high energies the $n$-particle contribution overtakes the $(n-2)$-particle contribution, i.e. $\rho^{(n)}(\mu)>\rho^{(n-2)}(\mu)$, for $\mu>\mu_{n}$, where $\rho^{(n)}\left(\mu_{n}\right)=\rho^{(n-2)}\left(\mu_{n}\right)$. (This feature is not directly visible in figures 1 and 2 because of the relative rescaling and the insufficient magnification of the intersection region.) The 


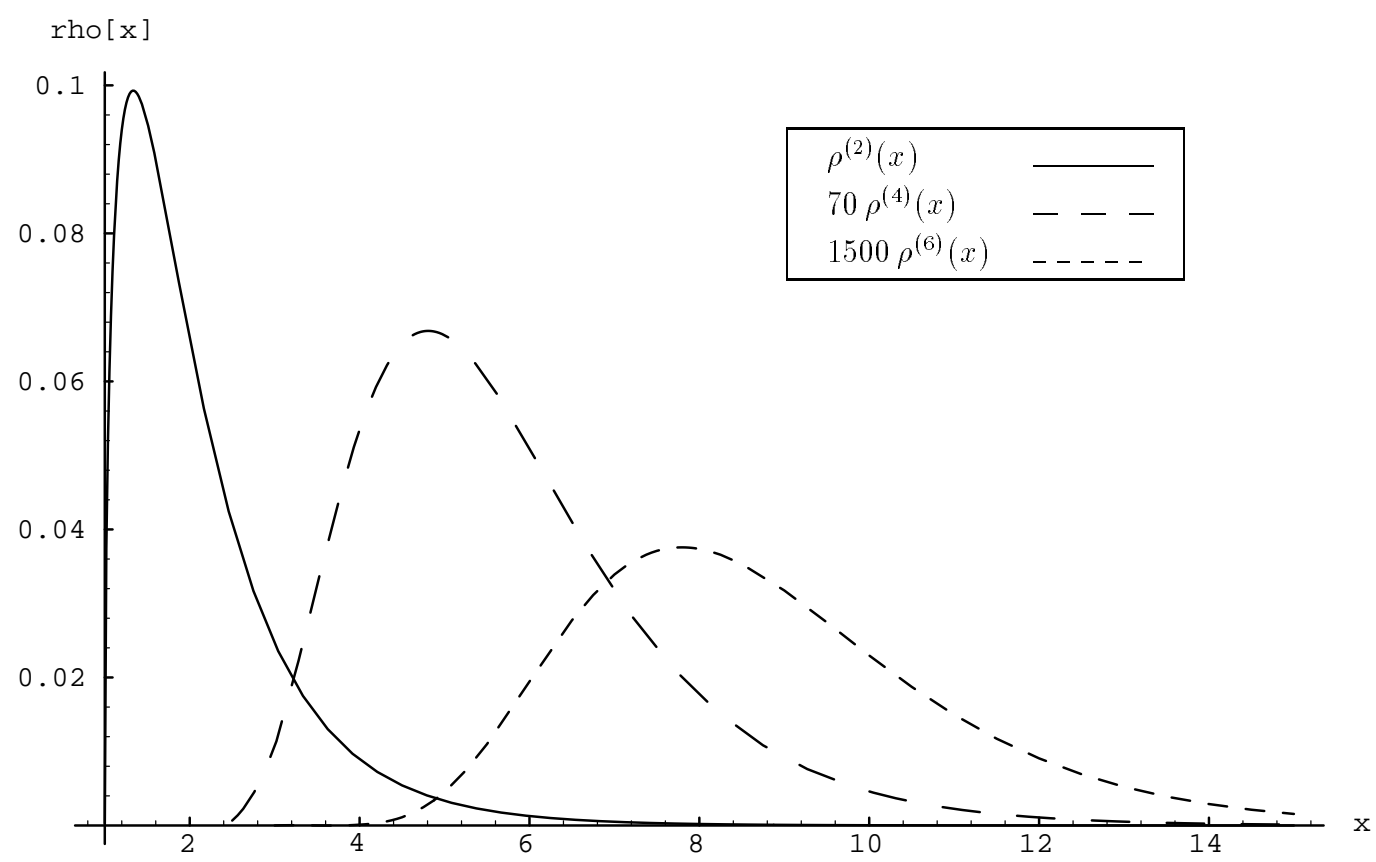

Figure 2: 2-, 4-, and 6-particle contribution to the spectral density of the current as a function of $x=\log _{2}(\mu / m)$.

point of intersection $\mu_{n}$ is of particular interest because it provides an intrinsic measure for the quality of the approximation made by truncating the form factor series at the $n$-particle term: Since $\mu_{n+2} \gg \mu_{n}$ the $(n+2)$-particle contribution can safely be ignored up to energies $\mu \lesssim \mu_{n}$ in the sense that the correction to $\rho^{(2)}(\mu)+\ldots+\rho^{(n)}(\mu)$ would be at most (of the order of) a percent. The results for these points of intersection $\left(\mu_{n}, \rho^{(n-2)}\left(\mu_{n}\right)=\rho^{(n)}\left(\mu_{n}\right)\right)$ are as follows:

$$
\begin{aligned}
\text { EM: } & n=4:\left(1.7 \cdot 10^{2}, 4.7 \cdot 10^{-6}\right), \quad n=6:\left(4.5 \cdot 10^{5}, 2.5 \cdot 10^{-10}\right), \\
\text { Current: } & n=4:\left(1.6 \cdot 10^{2}, 3.8 \cdot 10^{-4}\right), \quad n=6: \quad\left(1.2 \cdot 10^{6}, 3.2 \cdot 10^{-8}\right) .
\end{aligned}
$$

Thus, the form factor series truncated at 6 particles should provide accurate results for the spectral density up to energies $O\left(10^{5}\right)$ - results, which can then be compared with those obtained by other techniques.

In the high energy regime one expects the onset of asymptotic freedom and the perturbative predictions should coincide fairly well with the form factor curves. This is indeed the case as can be seen in figure 3. What is shown is the Fourier transform $I\left(p^{2}\right)$ of the 2-point function of the Noether current, computed once in 2-loop PT and once via (7) by truncation of the form factor series. In performing the integral transformation (7) one 
can see that accurate results for $\rho(\mu)$ in the region $2 \lesssim \mu \lesssim 10^{5}$ produce accurate results for $I(z)$ in the range $z \lesssim 10^{4}$. In the energy range $100 \lesssim \mu / m \lesssim 10^{4}$ the coincidence of the 2-loop PT curve and the $2+4+6$-particle form factor curve indicates that the system is desribed well by 2-loop PT in this regime. The result (17) for the intersection point $\mu_{6}$ suggests that the deviation at yet larger energies can entirely be attributed to the truncation of the form factor series. Notice however that the onset of the (2-loop) perturbative regime occurs at much higher energies $\mu / m \gtrsim 100$ than is sometimes pretended in the 4-dimensional counterpart of this situation. Figure 4 is a magnification of the low energy region of figure 3, where non-perturbative effects are expected to become important.

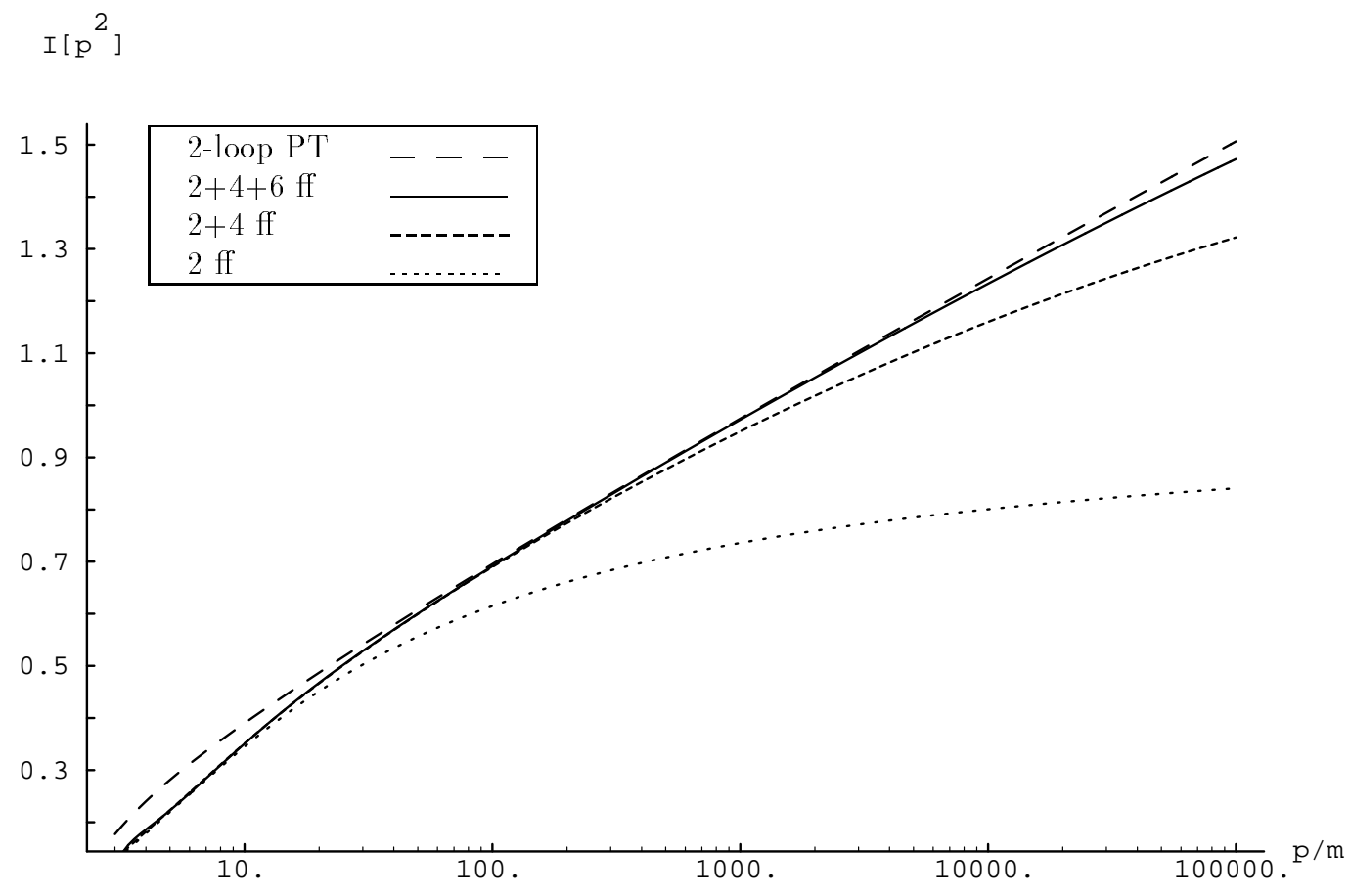

Figure 3: Comparison: Form factor approach versus 2-loop perturbation theory; logplot of $I\left(p^{2}\right)$ against $p / m$.

Here Monte Carlo simulations provide an alternative non-perturbative technique to probe the system [17, 7]. The simulations were made [7] using a Wolff-type cluster algorithm on a 460 square lattice at inverse coupling $\beta=1.80$ (correlation length $\xi=65.05$ ). The agreement between the $\mathrm{MC}$ data and the form factor results is excellent. One also sees that for energies between 30 and 45 the PT curve runs almost parallel to the MC data. Without the guidance of the form factor result one would thus be tempted to match both curves by tuning the Lambda parameter approriately. Doing this however, the 


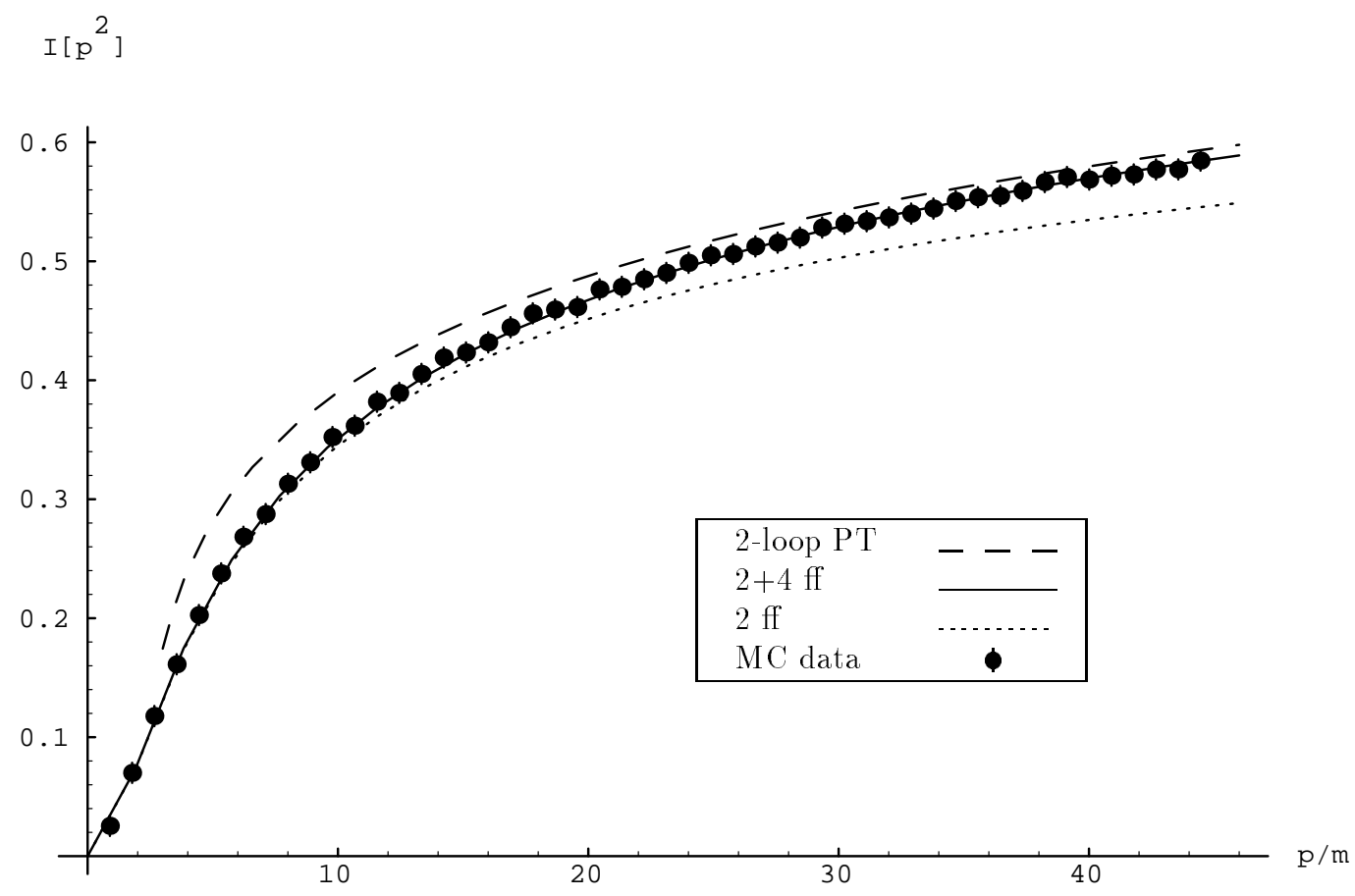

Figure 4: Low energy region of figure 3. Comparison: Form factor approach, Monte Carlo data and 2-loop perturbation theory; $I\left(p^{2}\right)$ plotted against $p / m$.

Lambda parameter comes out wrong by about 10\% (from below), as compared with the known exact result [8]. Generally speaking one sees that a determination of the Lambda parameter from MC data and (2-loop) PT about the onset of the (2-loop) perturbative regime enters. The form factor results in the $O(3)$-NLS model show that this regime sets in only at relatively high energies $\mu / m \gtrsim 100$, which should at least be taken as a warning in 4-dimensional gauge theories.

Acknowledgements: We wish to thank H. Lehmann, A. Patriasciou, E. Seiler and P. Weisz for stimulating discussions. A. P. and E. S. we thank in addition for generously allowing us to use their data prior to publication. M. N. acknowledges support by the Reimar Lüst fellowship of the Max Planck Society. T. H. acknowledges support by the D.O.E. (cooperative research agreement DE-FCO2-94ER40818). 


\section{References}

[1] M. Karowski and P. Weisz, Nucl. Phys. B139 (1978) 455

[2] F.A. Smirnov, 'Form Factors in Completely Integrable Models of Quantum Field Theory', World Scientific, 1992

[3] A. Zamolodchikov and Al.B. Zamolodchikov, Ann. Phys. 120 (1979) 233

[4] V. P. Yurov and Al. B. Zamolodchikov, Int. J. Mod. Phys. A6 (1991) 3419

Al. B. Zamolodchikov, Nucl. Phys. B348 (1991) 619

[5] A. Fring, G. Mussardo and P. Simonetti, Nucl. Phys. B393 (1993) 413

J. L. Cardy and G. Mussardo, Nucl. Phys. B410 (1993) 451

G. Delfino and G. Mussardo, Phys. Lett. B324 (1994) 40

G. Delfino and G. Mussardo, Nucl. Phys. B455 (1995) 724

C. Acerbi, G. Mussardo and A. Valleriani, hep-th/9601113

[6] H. Lesage, H. Saleur and S. Skorik, cond-mat/9603043

[7] A. Patriasciou and E. Seiler, to be published

[8] P. Hasenfratz, M. Maggiore and F. Niedermayer, Phys. Lett. B245 (1990) 522

P. Hasenfratz and F. Niedermayer, Phys. Lett. B245 (1990) 529

[9] A. Zamolodchikov, JETP Lett. 43 (1986) 731

[10] A. Capelli, D. Friedan and J.L. Latorre, Nucl. Phys. B352 (1991) 616

[11] A.N. Kirillov and F.A. Smirnov, Phys. Lett. B198 (1987) 506; Int. J. Mod. Phys. A3 (1988) 731

[12] J. Balog and M. Niedermaier, in preparation

[13] A. Patriasciou and E. Seiler, 'Two dimensional NLS Models: Orthodoxy and Heresy', in Fields and Particles, Schladming 1990, ed. H. Mitter and W. Schweiger

[14] J. Balog, Phys. Lett. B300 (1993) 145

[15] J. Balog and T. Hauer, Phys. Lett. B337 (1994) 115

[16] V.A. Fateev and Al.B. Zamolodchikov, Phys. Lett. B271 (1991) 91

[17] M. Lüscher and U. Wolff, Nucl. Phys. B339 (1990) 222 